\title{
Does Focal Nodular Hyperplasia Predispose to Metastatic Growth of Renal Cell Carcinoma-A Surgical Dilemma
}

\author{
Tympa Aliki ${ }^{1}$, Koutoulidis Vasilios ${ }^{2}$, Dellaportas Dionysios ${ }^{3}$, Nastos Constantinos ${ }^{3}$, \\ Psychogiou Vasiliki ${ }^{3} \&$ Smyrniotis Vasilios ${ }^{4}$ \\ ${ }^{1}$ Department of Anesthesiology, University of Athens, Aretaieio Hospital, Athens, Greece \\ ${ }^{2}$ Department of Radiology, University Hospital Aretaieion, Greece \\ ${ }^{3}$ Department of Surgery, University of Athens, Aretaieio Hospital, Greece \\ 4 Department of Surgery, University of Athens, Attikon Hospital, Greece \\ Correspondence: Dellaportas Dionysios, Department of Surgery, University of Athens, Aretaieio Hospital, 76 \\ Vas. Sofias Ave., 11576, Athens, Greece. Tel: 30-694-771-8723. E-mail: dellapdio@gmail.com
}

Received: December 11, $2011 \quad$ Accepted: December 26, $2012 \quad$ Online Published: May 1, 2012
doi:10.5539/cco.v1n1p133
URL: http://dx.doi.org/10.5539/cco.v1n1p133

The authors declare that they have no competing interests

\begin{abstract}
Introduction: Focal nodular hyperplasia is a benign liver disease usually treated conservatively. However, in the setting of a previously resected renal cell carcinoma (RCC) a liver mass with imaging characteristics of focal nodular hyperplasia (FNH) raises a surgical dilemma. Case report: Four years after an initial right nephrectomy for stage I RCC and concurrent oncocytoma, a 72 year-old Caucasian male presented with a liver lesion. Imaging studies raised a question of differential diagnosis between FNH and metastatic RCC. Given the facts, firstly that the radiological findings were suggestive of $\mathrm{FNH}$, secondly that the lesion of the liver was a new finding four years post-resection of the RCC and third that co-existence of FNH and metastatic disease have been reported but the age and sex of the patient were not compatible with the epidemiology of FNH the patient underwent liver resection. Histopathological findings were consistent with FNH and nests of malignant cells compatible with RCC were found in the lesion.
\end{abstract}

Keywords: focal nodular hyperplasia; renal cell carcinoma; metastasis

\section{Introduction}

Focal nodular hyperplasia (FNH) is the second most common benign liver disease. It usually occurs in women of childbearing and middle age and treatment is conservative. However, in the setting of a previous renal cell carcinoma (RCC), a liver mass with imaging characteristics of FNH raises the question of surgical intervention, given the fact that metastatic disease or FNH harboring malignant cells cannot be ruled out. The previous statement is based on three such cases reported in the past and our case also. We present a rare case of FNH, which harbored nest of malignant metastatic cells in a patient with a history of resected synchronous renal cell carcinoma and oncocytoma. The pathologic findings along with the surgical dilemma are also discussed.

\section{Case Report}

Four years after an initial right nephrectomy for stage I renal cell carcinoma (RCC), which measured 5,2 cm and concurrent oncocytoma, a 72 year-old Caucasian male presented with a liver lesion. The abdominal computed tomography (CT) scan demonstrated a $4 \mathrm{~cm}$ mass in the right liver lobe (segment VIII), with malignant characteristics. The radiological diagnosis was highly suspicious for metastatic RCC (Figure 1). Liver function tests and tumor markers were within normal range. A supplementary abdominal magnetic resonance imaging (MRI) confirmed the $4 \mathrm{~cm}$ mass in the right liver lobe and raised a question of differential diagnosis between FNH and metastatic RCC (Figure 2). Regular CT scans for a period up to four years, after the initial diagnosis, had not shown any pathologic lesions. Surgical intervention was considered to be the treatment of choice, since the differential diagnosis included resectable RCC liver metastasis or FNH which might harbor malignant cells. A liver resection was performed with the patient having an uneventful recovery and being discharged on the seventh postoperative day. The resected segment of the liver weighed 706g. On sectioning, a circumscribed 
yellow-tan tumor, measuring $4 \times 3 \times 2,5 \mathrm{~cm}$ with nodular cut surface was revealed. Microscopically the lesion was multinodular and had connective tissue septae containing abundant dilated, thin-walled vessels (Figure 3 ). There was ductular proliferation at the periphery of the nodules (Figure 4). These findings were consistent with FNH. Also, nests of malignant cells compatible with RCC where found within tissue septae.

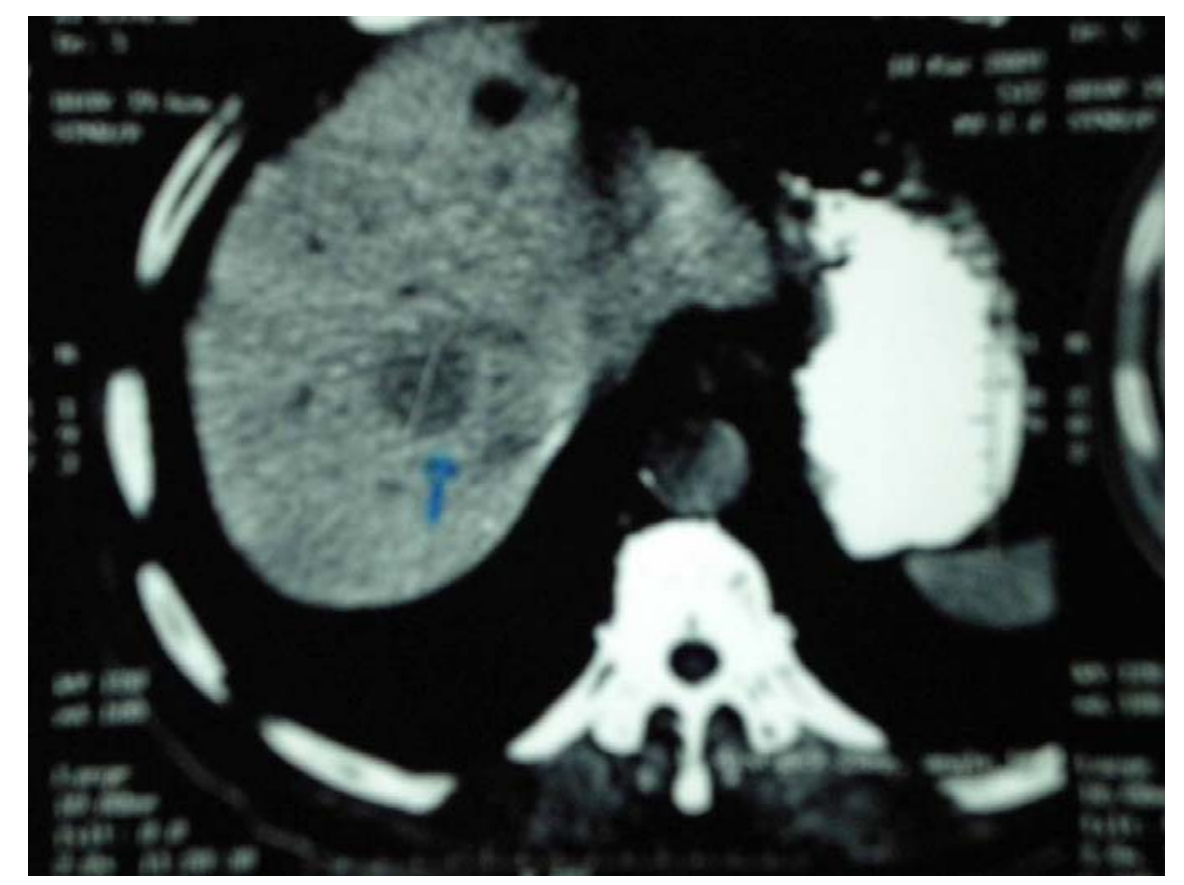

Figure 1. Abdominal CT-scan which demonstrates a $4 \mathrm{~cm}$ mass in the right liver lobe

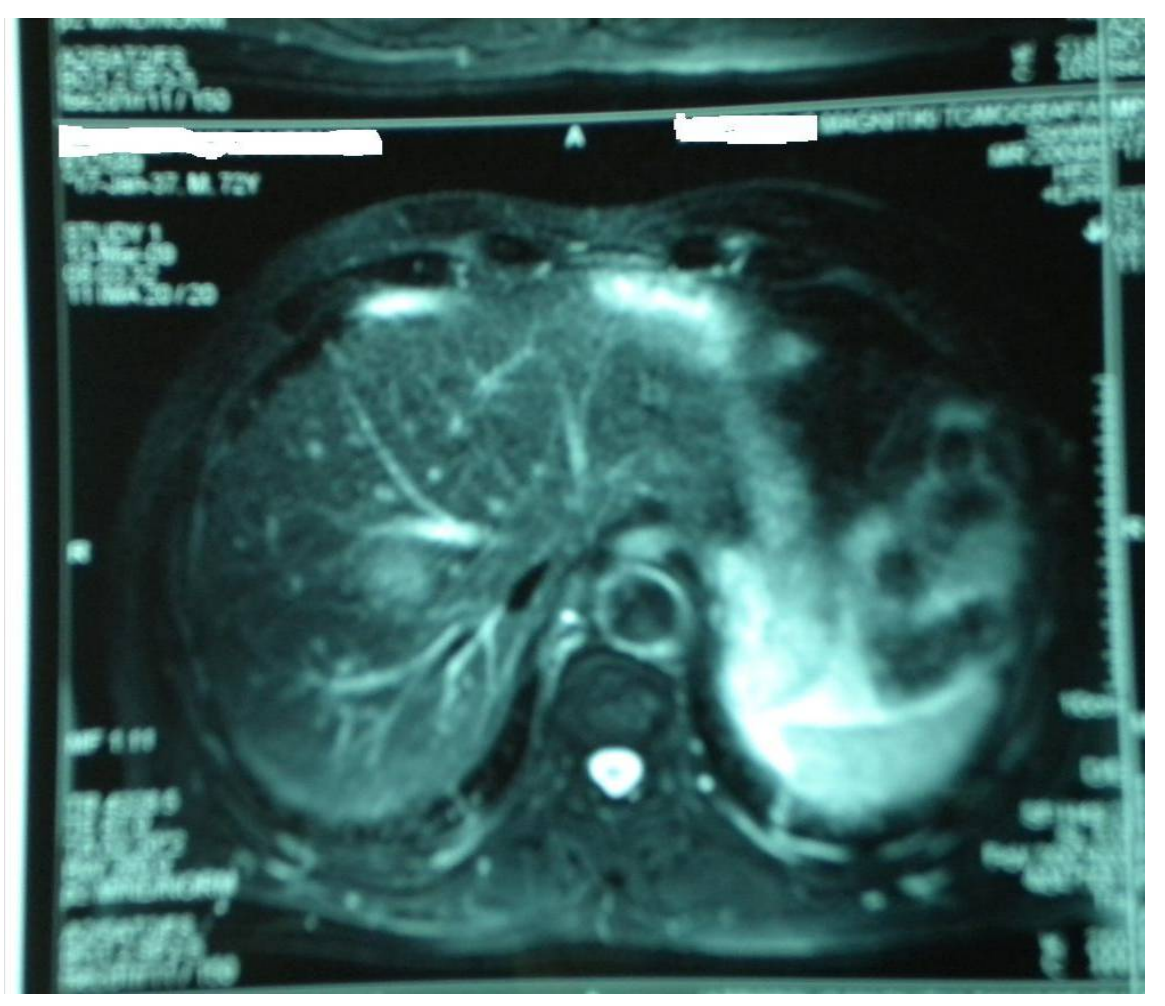

Figure 2. MRI-scan demonstrating the liver mass 


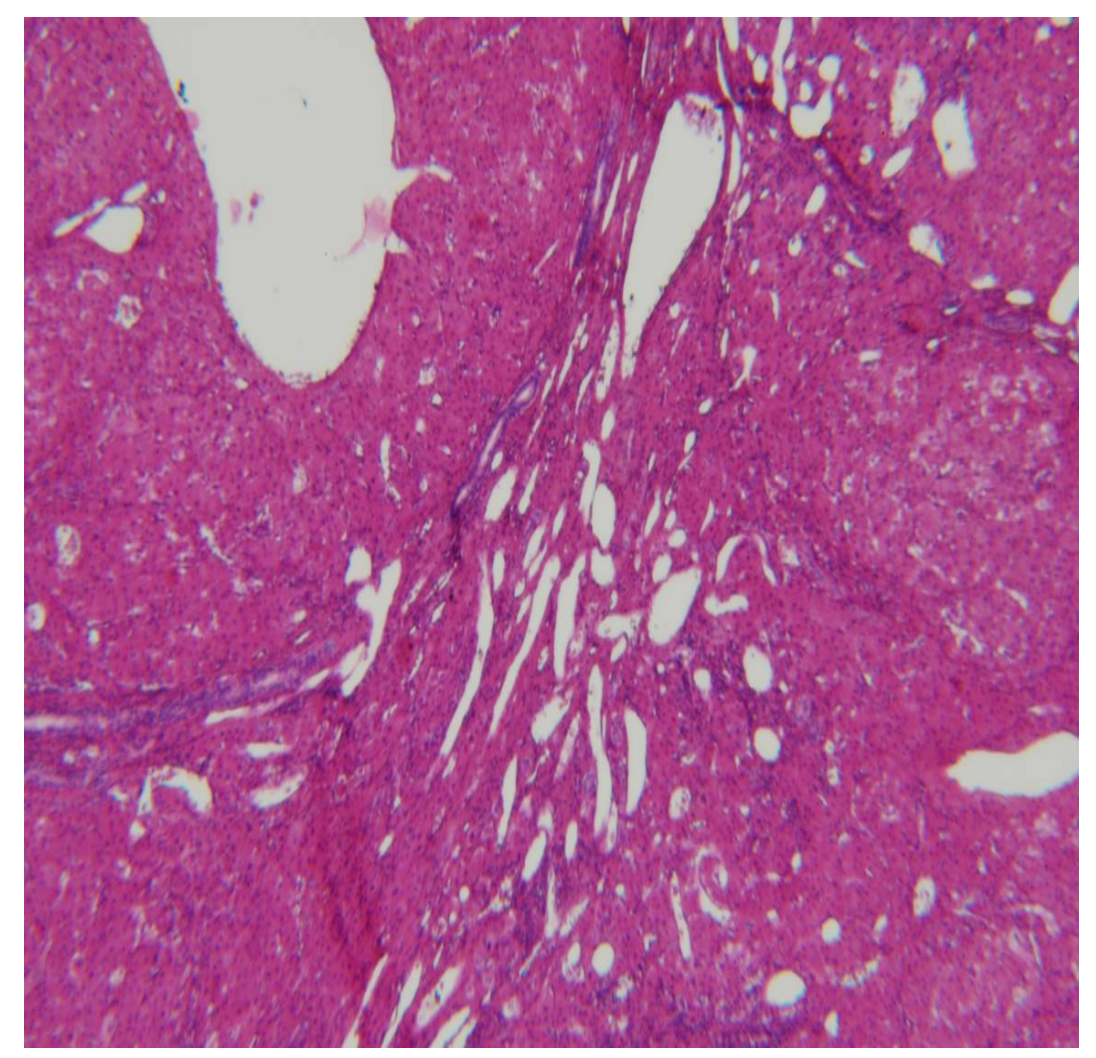

Figure 3. The lesion has multinodular pattern. Several dilated, thin-walled vessels are seen in the fibrous septae

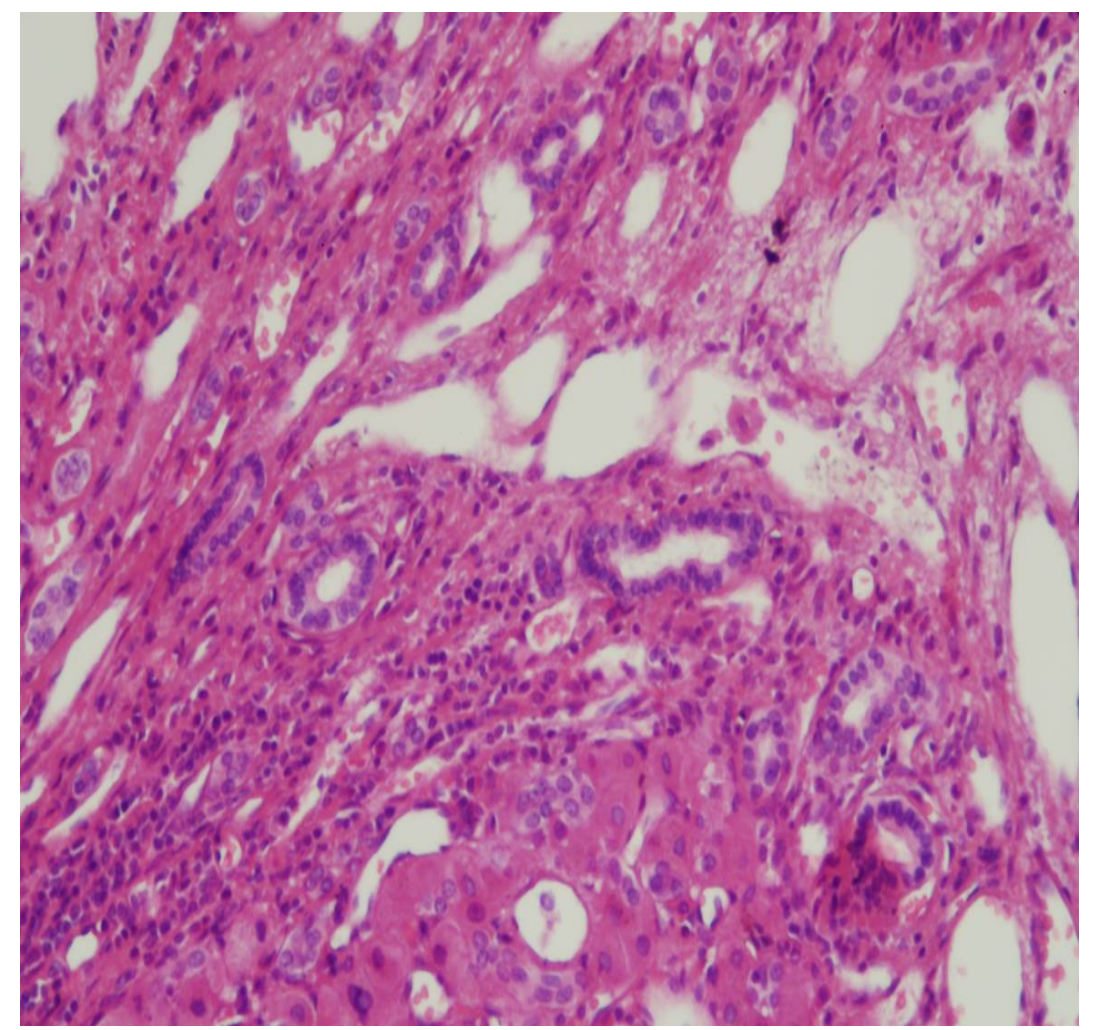

Figure 4. Ductular proliferation at the periphery of the nodules 


\section{Discussion}

Focal nodular hyperplasia is the second most common benign liver disease after hemangioma. The female to male ratio, is between 6:1 and 8:1. Mean age of diagnosis is 40 years. Although a causal relationship has not been established, oral contraception seems to induce FNH (Mathieu et al., 2000). It appears that symptoms of FNH are non-specific. Patients most often complain of an abdominal mass or abdominal pain. In many cases, FNH is an incidental finding during upper abdominal imaging tests. On non-contrast CT, FNH appears hypointense or isointense. Following contrast medium injection, during the arterial phase, a rapid enhancement of the lesion is observed. Minor enhancement persists in the portal venous phase. The lesion becomes hypo- or iso-intense. On the other hand the central scar of the tumor is hypointense on arterial phase and hyperintense on the portal venous phase, due to delayed washout (Mathieu et al., 1997). FNH is most frequently found in the right hepatic lobe and its gross appearance is characteristic: it is well circumscribed but not encapsulated and has a lobulated cut surface with a central branching scar.

On the other hand, metastatic liver disease usually presents as a hypointense mass before the administration of the contrast medium. Moreover, metastatic liver masses are intensely enhanced in the arterial phase and hypointense during the portal venous phase, since their blood supply is the hepatic artery(Fulcher \& Sterling, 2002). The distinction between metastatic liver disease and FNH is uncertain and benign liver lesions can be misdiagnosed as metastatic renal cell carcinoma (Wishnow, Charnsangavej, \& Babaian, 1989).

FNH is considered to be a non-neoplastic, hyperplastic response to a congenital venous dysplasia rather than an hamartoma (Wanless, Mawdsley, \& Adams, 1985). The above mentioned dysplasia correlates with the increased blood flow to this benign tumor, which in turn could grow into a host lesion for a tumor to tumor metastasis. In a brief review of the literature three cases of such a co-existence have been reported before. The first one is a case of a metastatic RCC within a FNH (Wheeler, Wheeler, Diaz-Arias, \& Anders, 2009), and the second is a case of metastatic rectal adenocarcinoma associated with FNH (Nisar et al., 2002). The third case which correlates to our case, refers to a patient with Von-Hippel-Lindau disease who had metastatic deposit of a primary RCC within a FNH (Dogrul et al., 2009). Wheeler et al, who reported the first case, propose two theories in order to explain this rare co-existence. Firstly, high blood flow to the FNH predisposes the region to develop a metastatic neoplasm from the systemic, non-portal circulation. Secondly, it is possible that metastatic tumor may cause a local vascular flow abnormality, which induces hepatocellular hyperplasia, resulting in secondary co-existing FNH (Bioulac-Sage, Balabaud, \& Wanless, 2001).

FNH in a patient with history of malignancy warrants awareness. Malignant elements within the lesion cannot easily be excluded by advanced imaging techniques.

Despite the fact that in our patient the imaging characteristics were diagnostic of FNH, we opted for surgical resection of the lesion for the following reasons:

1) Radiological findings were suggestive of $\mathrm{FNH}$, however, not pathognomonic,

2) The lesion of the liver was a new finding four years after the resection of RCC,

3) Co-existence of FNH and metastatic disease have been reported but the age and sex of the patient were not compatible with the epidemiology of FNH. Finally, our resection specimen confirmed the malignant metastatic host potential of such a liver lesion.

\section{Conclusion}

In conclusion FNH is a benign liver disease and no surgery is needed in most cases. However, for patients with previous or concurrent malignancies the possibility of an FNH lesion to host malignant cells may be an emerging indication for operative management of FNH.

\section{Consent}

Written informed consent was obtained from the patient for publication of this case report and accompanying images. A copy of the written consent is available for review by the Editor-in-Chief of this journal. 


\section{References}

Bioulac-Sage, P., Balabaud, C., \& Wanless, I. R. (2001). Diagnosis of focal nodular hyperplasia: not so easy. Am J Surg Pathol, 25(10), 1322-1325. http://dx.doi.org/10.1097/00000478-200110000-00015

Dogrul, A. B., Erol, T., Karakoc, D., Hamaloglu, E., Ozdemir, A., \& Ozenc, A. (2009). Renal cell carcinoma metastasis to focal nodular hyperplasia focus on liver in a patient with Von Hippel-Lindau disease. Am J Med Sci, 338(2), 159-160. http://dx.doi.org/10.1097/MAJ.0b013e3181a02b2b

Fulcher, A. S., \& Sterling, R. K. (2002). Hepatic neoplasms: computed tomography and magnetic resonance features. J Clin Gastroenterol, 34(4), 463-471. http://dx.doi.org/10.1097/00004836-200204000-00019

Mathieu, D., Kobeiter, H., Maison, P., Rahmouni, A., Cherqui, D., Zafrani, E. S., \& Dhumeaux, D. (2000). Oral contraceptive use and focal nodular hyperplasia of the liver. Gastroenterology, 118(3), 560-564. http://dx.doi.org/10.1016/S0016-5085(00)70262-9

Mathieu, D., Vilgrain, V., Mahfouz, A. E., Anglade, M. C., Vullierme, M. P., \& Denys, A. (1997). Benign liver tumors. Magn Reson Imaging Clin N Am, 5(2), 255-288.

Nisar, P. J., Zaitoun, A. M., Damera, A., Hodi, Z., Tierney, G. M., \& Beckingham, I. J. (2002). Metastatic rectal adenocarcinoma to the liver associated with focal nodular hyperplasia. J Clin Pathol, 55(12), 967-969. http://dx.doi.org/10.1136/jcp.55.12.967

Wanless, I. R., Mawdsley, C., \& Adams, R. (1985). On the pathogenesis of focal nodular hyperplasia of the liver. Hepatology, 5(6), 1194-1200. http://dx.doi.org/10.1002/hep.1840050622

Wheeler, Y. Y., Wheeler, G. L., Diaz-Arias, A. A., \& Anders, R. A. (2009). Metastatic renal cell carcinoma within a hepatic focal nodular hyperplasia: a case report and review of the literature. Int J Clin Exp Pathol, 2(2), 190-193.

Wishnow, K. I., Charnsangavej, C., \& Babaian, R. J. (1989). Benign hepatic masses mimicking metastatic renal cell carcinoma. Urology, 33(3), 250-252. http://dx.doi.org/10.1016/0090-4295(89)90405-6 